\title{
The golden 35 min of stroke intervention with ADAPT: effect of thrombectomy procedural time in acute ischemic stroke on outcome
}

\author{
Ali Alawieh, ${ }^{1}$ Alyssa K Pierce, ${ }^{2}$ Jan Vargas, ${ }^{2}$ Aquilla S Turk, ${ }^{2}$ Raymond D Turner, ${ }^{2}$ \\ M Imran Chaudry, ${ }^{2}$ Alejandro M Spiotta ${ }^{2}$
}

${ }^{1}$ Medical Scientist Training Program, Medical University of South Carolina, Charleston, South Carolina, USA

${ }^{2}$ Department of Neurosurgery, Medical University of South Carolina, Charleston, South Carolina, USA

Correspondence to Dr Alejandro M Spiotta, Department of Neurosciences, Division of Neurosurgery, Medical University of South Carolina, 96 Jonathan Lucas Street, CSB 210, Charleston, SC 29425, USA; spiotta@musc.edu

Received 16 February 2017 Revised 24 March 2017 Accepted 25 March 2017 Published Online First 2 May 2017

Check for updates

To cite: Alawieh $A$ Pierce AK, Vargas J, et al. J Neurolntervent Surg 2018:10:213-220.
ABSTRACT

Introduction In acute ischemic stroke (AIS), extending mechanical thrombectomy procedural times beyond 60 min has previously been associated with an increased complication rate and poorer outcomes.

Objective After improvements in thrombectomy methods, to reassess whether this relationship holds true with a more contemporary thrombectomy approach: a direct aspiration first pass technique (ADAPT).

Methods We retrospectively studied a database of patients with AIS who underwent ADAPT thrombectomy for large vessel occlusions. Patients were dichotomized into two groups: 'early recan', in which recanalization (recan) was achieved in $\leq 35 \mathrm{~min}$, and 'late recan', in which procedures extended beyond $35 \mathrm{~min}$.

Results 197 patients (47.7\% women, mean age 66.3 years) were identified. We determined that after $35 \mathrm{~min}$, a poor outcome was more likely than a good (modified Rankin Scale (mRS) score 0-2) outcome. The baseline National Institutes of Health Stroke Scale (NIHSS) score was similar between 'early recan' $(n=122)$ $(14.7 \pm 6.9)$ and 'late recan' patients $(n=75)(15.9 \pm 7.2)$. Among 'early recan' patients, recanalization was achieved in $17.8 \pm 8.8 \mathrm{~min}$ compared with $70 \pm 39.8 \mathrm{~min}$ in 'late recan' patients. The likelihood of achieving a good outcome was higher in the 'early recan' group $(65.2 \%)$ than in the 'late recan' group (38.2\%; $\mathrm{p}<0.001)$. Patients in the 'late recan' group had a higher likelihood of postprocedural hemorrhage, specifically parenchymal hematoma type 2 , than those in the 'early recan' group. Logistic regression analysis showed that baseline NIHSS, recanalization time, and atrial fibrillation had a significant impact on 90-day outcomes.

Conclusions Our findings suggest that extending ADAPT thrombectomy procedure times beyond $35 \mathrm{~min}$ increases the likelihood of complications such as intracerebral hemorrhage while reducing the likelihood of a good outcome.

\section{INTRODUCTION}

Current interventions to restore blood flow after acute ischemic stroke (AIS) include administration of systemic IV tissue plasminogen activator (IV tPA), IA thrombolysis, and mechanical thrombectomy. ${ }^{1-5}$ Randomized controlled trials on endovascular interventions, including balloon angioplasty, IA thrombolysis, stenting, aspiration, and mechanical maceration, have shown that these interventions can reduce the severity of AIS with an extended treatment window beyond 8 hours of onset. $^{3-21}$

Despite the type of acute treatment, studies on AIS have focused on the time from symptom onset to treatment when evaluating outcomes. We have previously determined that, in the case of mechanical thrombectomy, procedural time, rather than time from onset to treatment, may have a stronger impact on outcomes in these patients. In particular, procedures that extended beyond $60 \mathrm{~min}$ from groin puncture to recanalization resulted in increased incidence of complications, increased cost, and worse outcomes. ${ }^{22}$ Mechanical thrombectomy in this study was performed using Penumbra separators and, less frequently, stent retrievers. ${ }^{22}$ Following this study, the introduction of a direct aspiration at first pass technique (ADAPT) with large bore aspiration catheter has significantly reduced the time required to achieve satisfactory angiographic outcomes. ${ }^{23}{ }^{24}$ ADAPT has been shown to be a fast and effective method for achieving thrombectomies with good clinical and angiographic outcomes while reducing procedure time to around $30 \mathrm{~min}$, on average. ${ }^{23} 24$ The purpose of this study is to re-evaluate, using ADAPT, if a similar relationship between procedural time and outcome still holds true, and whether further reduction in procedure time to within the first half hour results in better outcomes.

\section{METHODS}

\section{Patient selection}

We retrospectively studied a database of patients with AIS involving the anterior circulation who underwent ADAPT thrombectomy at a tertiary care center between December 2012 and July 2015. Candidacy for intervention was determined by CT perfusion imaging, irrespective of time of onset. ${ }^{25}$ The study was approved by the institutional review board. Every patient underwent postprocedural CT within 12-24 hours routinely and the presence of hemorrhage on CT was determined by a blinded neuroradiologist. In cases where there was question of contrast staining versus petechial hemorrhage, subsequent CT scans were reviewed to assess for washout of contrast. If the follow-up imaging did not clarify contrast staining versus petechial hemorrhage, petechial hemorrhage was assumed. None of the patients required a decompressive craniotomy. ADAPT has been described previously. ${ }^{23} 24$ For device selection, the largest caliber aspiration 
catheter that the vessel can accommodate is selected. For M1 thrombi, the 5 MAX, 5 MAX ACE, 064 and 068 catheters were primarily employed. In smaller caliber or more distal vessels, the technique is employed with either a 4 Max or 3 Max reperfusion catheter (Penumbra, Alameda, California, USA). A maximum of two to three attempts are performed with ADAPT before the use of additional devices such as stent retrievers at the discretion of the operator. All patients received conscious sedation during the procedure, and a permissive hypertensive blood pressure was maintained during procedures to allow collaterals, followed by blood pressure control according to standard institutional protocols.

\section{Data collection}

Patient data were collected by reviewing patient charts, procedural records, and angiograms, and included preprocedural, procedural, and outcome variables. Preprocedural variables were age, gender, initial National Institutes of Health Stroke Scale (NIHSS) score, comorbidities, time of symptom onset, whether IV tPA was given and whether only proximal vessels were involved. Procedural variables included recanalization status of the affected vessel, as determined by the Thrombolysis in Cerebral Infarction (TICI) scale, intraprocedural complications, time to recanalization, the total number of passes, the number of passes with ADAPT only, and the presence of hemorrhage on a postprocedural head CT scan. Hemorrhage was classified using the European Cooperative Acute Stroke Study radiological classification for hemorrhagic infarcts (HI1 and HI2), parenchymal hematomas (PH1 and PH2), and subarachnoid hemorrhage. ${ }^{26}$

For parts of the statistical analysis, time to recanalization was studied as a continuous variable. Time to recanalization was determined from percutaneous groin access (time zero) to the time that the affected vessel was recanalized. Time was monitored in minutes and seconds based on review of the time stamp of the angiographic images. If the patient had an intraprocedural complication requiring abortion of the procedure (eg, vessel rupture), recanalization time was defined as time of groin puncture to the time when the complication was documented. Patients were dichotomized into two groups: 'early recan', in which recanalization was achieved in $\leq 35 \mathrm{~min}$, and 'late recan', in which procedures extended beyond $35 \mathrm{~min}$. A duration of $35 \mathrm{~min}$ was chosen as a cut-off point since the curves representing the percentage of patients with good and poor outcomes intersect at around $35 \mathrm{~min}$, denoting the procedure time above which a poor outcome becomes more likely. A good outcome was defined as a modified Rankin Scale (mRS) score 0-2 and a poor outcome as an mRS score 3-6.

\section{Outcomes}

Functional outcome was assessed by 90-day follow-up mRS score, 90-day NIHSS score, and length of stay.

\section{Statistical analysis}

Statistical analyses were performed using SAS V.9.4 (SAS Institute, Cary, North Carolina, USA), Graphpad Prism 6 (Graphpad, La Jolla, California, USA). A population of 197 subjects was analyzed using descriptive statistics to characterize demographics and other clinical variables describing treatment, complications, and outcomes. Differences between the recanalization time groups ('early recan' and 'late recan') based on these variables were tested using Student's t test for continuous measures and a $\chi^{2}$ test for categorical measures. Differences between the device groups were tested using Fisher's exact test for categorical measures with expected cell sizes $<5$. All tests were two-sided and assessed at a significance level of 0.05 . Multivariate logistic regression was used to predict the outcome mRS score at 90 days of $0-2$ based on several prespecified prognostic variables, including time to recanalization. Model performance was assessed using the Hosmer-Lemeshow test and $c$ statistic.

A logistic regression analysis was performed to determine if the probability of a good outcome at 90 days was affected by prespecified prognostic variables. One hundred and forty-eight subjects were included in the model, of whom 84 subjects $(57 \%)$ had a good outcome. Seventeen subjects with a missing outcome and 32 subjects with missing values for a prognostic variable (onset to groin) were excluded from the model. The variables included in the multivariate model were recanalization time (as a categorical variable of 'early recan' ( $\leq 35 \mathrm{~min}$ ), baseline NIHSS score, revascularization (post-TICI flow of $2 b$ or 3 ), presence of hemorrhage or procedural complications, time from symptom onset to CT in hours, time from CT to procedure start in hours, treatment with IA or IV tPA, age, and indicators of atrial fibrillation, diabetes, hypertension, and number of passes.

For analyses shown in figure 2, a third-degree polynomial best-fit curve was determined using Graphpad prism based on the highest correlation coefficient $\left(\mathrm{R}^{2}\right)$ and compared with other models of fitting. Runs test was used to assess for significant deviation from actual data. A Kruskal-Wallis test with Dunn's multiple comparisons test was used to compare the difference in the number of vessels involved at different procedure durations (figure 2B), and the best fit for linear regression was used to determine the correlation between the incidence of PH2-type hemorrhage and procedure time.

\section{RESULTS}

\section{Patient baseline characteristics}

A total of 197 patients (mean age $66.3 \pm 14,45.7 \%$ female) underwent ADAPT thrombectomy for AIS during the study period. The average NIHSS at presentation was $15.2 \pm 7$, and average time from symptom onset to groin puncture was 7.9 \pm 6.1 hours (table 1 ). The mean NIHSS score was similar

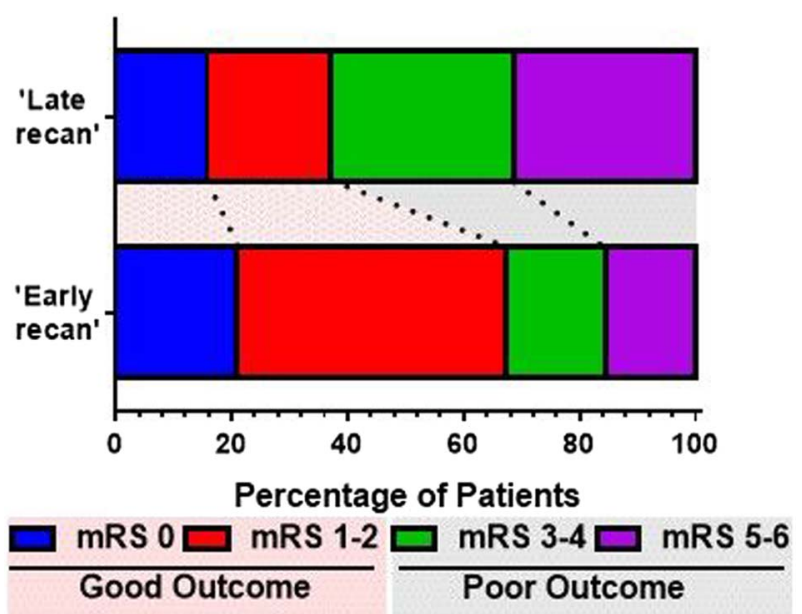

Figure 1 Distribution of 90-day modified Rankin Scale (mRS) outcome. Patients in the 'late recan' group (recanalization time $>35 \mathrm{~min}$ ) were more likely to have poor outcomes (mRS>2), and had a higher percentage of patients who had either died or were in a persistent vegetative state compared with 'early recan' group (recanalization time $\leq 35$ ). 
between 'early recan' $(14.7 \pm 6.9)$ and 'late recan' $(15.9 \pm 7.2$, $\mathrm{p}=0.207)$ groups. The two groups also had similar rates of comorbidities, including atrial fibrillation, diabetes and hypertension, rate of preprocedural IV tPA administration, rate of only proximal vessel involvement, and average time from symptom onset to groin puncture $(p>0.05)$. Patients in the 'early recan' group were more likely to be younger than those in the 'late recan' group $(64.2 \pm 14.1$ vs $69.9 \pm 13.3, \mathrm{p}=0.005)$.

\section{Recanalization}

Mean time for recanalization was $36.9 \pm 34.2$ min among all patients, and was significantly longer in the 'late recan' group
$(68.4 \pm 38.4 \mathrm{~min}$ vs $18.4 \pm 8.4 \mathrm{~min}, \mathrm{p}<0.001)$. Final TICI flow $\geq 2 \mathrm{~b}$ was achieved in $96.4 \%$ of patients $(n=190)$, TICI 3 was achieved in $59.4 \%$ of patients $(n=117), 2 \mathrm{c}$ in $8.1 \% \quad(n=16)$ and $2 \mathrm{~b}$ in $28.9 \%(\mathrm{n}=57)$. Patients in the 'early recan' group were more likely to achieve a TICI $\geq 2$ b (99.2\% vs $92.0 \%$, $\mathrm{p}=0.013)$.

Devices used in the ADAPT procedure are primarily the largest caliber aspiration catheter that the vessel can accommodate, including 3 MAX, 4 MAX, 5 MAX, 5 MAX ACE, 064 and 068 catheters. Upon failure of ADAPT, additional devices, including stent retrievers and carotid stents, are used. No balloon-guide catheters were used in these procedures.

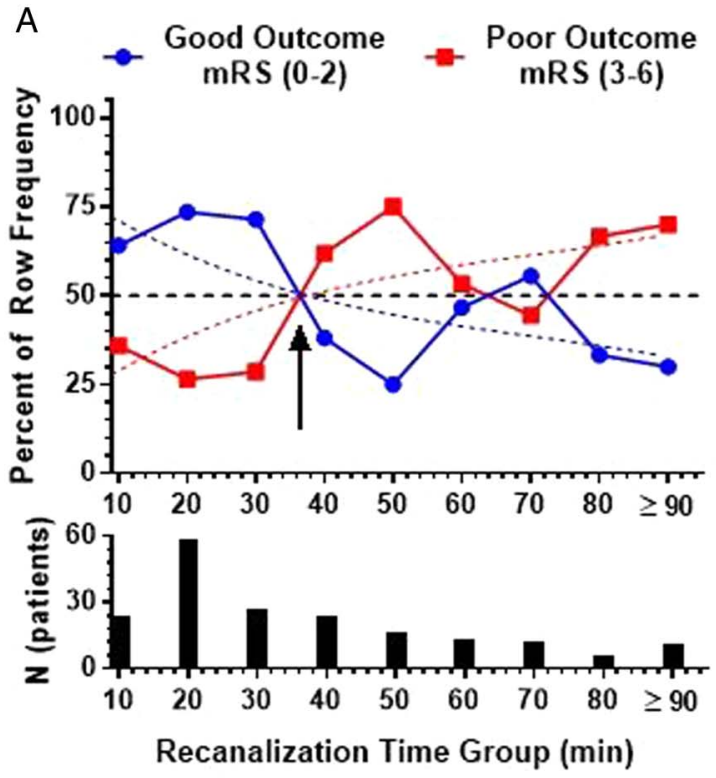

B

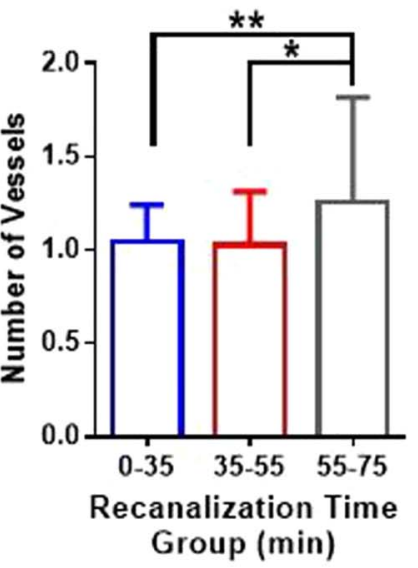

C Incidence of Hemorrhage (PH2) as a Function of Recanalization Time

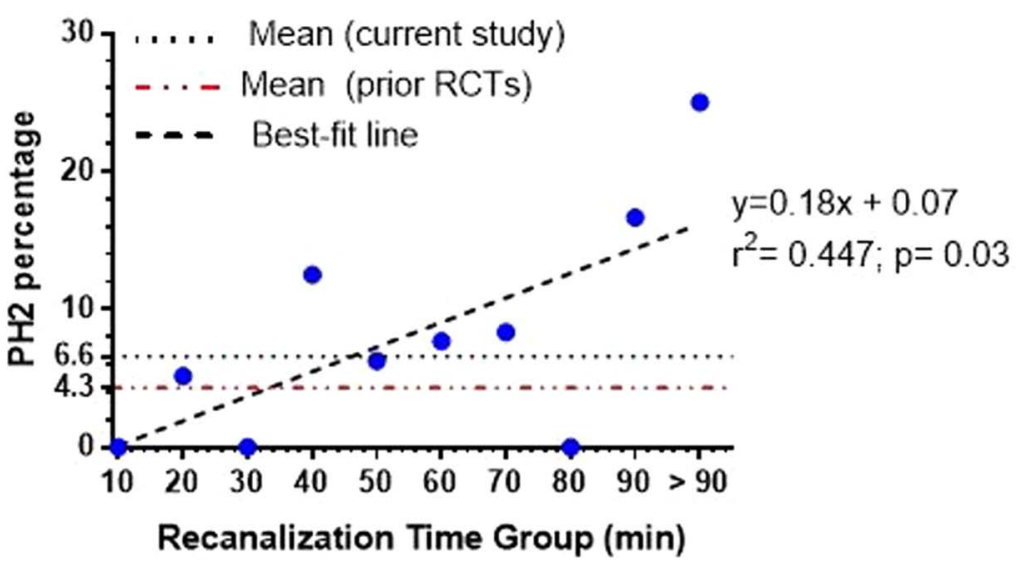

Figure 2 Outcomes at 90 days by recanalization time group. (A) The distribution of patients by good and poor outcomes and the number of patients at different recanalization time groups. Solid lines connect the actual data points for the distribution of percentage of good outcomes (blue) and bad outcomes (red). Dotted lines show the best fit curve for the distribution of percentage of good outcomes (blue) and bad outcomes (red). The arrow shows the point of intersection of curves with the $50 \%$ line denoting equal probability of good and bad outcomes. Best-fit curves are third-order polynomials with $R^{2}=0.54$. Deviation from the curves was not significant (runs test; $p=0.41$ ). (B) Comparison of the number of vessels occluded in the 55-75 min recanalization time group with the $0-35 \mathrm{~min}$ and 35-55 min groups, showing significantly higher number of vessels occluded in the 55-75 min group than in the other two groups. There was no significant difference in the number of vessels occluded in the 0 35 min and 35-55 min groups. A Kruskal-Wallis test was used with Dunn's multiple comparisons; ${ }^{*} p<0.05 ;{ }^{*} p<0.01$. (C) Correlation between percentage incidence of parenchymal hematoma (PH)2-type hemorrhage and recanalization time showing a significant positive correlation (Pearson's correlation, $\mathrm{R}^{2}=0.447, \mathrm{p}=0.03$ ), indicating a higher incidence of $\mathrm{PH} 2$-type hemorrhage with increased recanalization time. The incidence of $\mathrm{PH}$-type hemorrhage with recanalization time beyond 35 min was higher than the average of this study (6.6\%) and higher than that found in previous randomized controlled trials (RCTs) using different interventions $(4.3 \%){ }^{4}{ }^{18-21}$ 
Ischemic stroke

Table 1 Patient characteristics

\begin{tabular}{|c|c|c|c|c|c|}
\hline Variable & No of patients & Total & $\begin{array}{l}\text { 'Early recan' } \\
\leq 35 \mathrm{~min}\end{array}$ & $\begin{array}{l}\text { 'Late recan' } \\
>35 \mathrm{~min}\end{array}$ & p Value \\
\hline \multicolumn{6}{|l|}{ Preprocedure } \\
\hline Age (years) & 197 & $66.3(14)$ & $64.2(14.1)$ & $69.9(13.3)$ & 0.005 \\
\hline Female, n (\%) & 197 & $90(45.7)$ & $55(45.1)$ & $35(46.7)$ & 0.828 \\
\hline White, $n(\%)$ & 197 & $112(56.9)$ & $67(54.9)$ & $45(60)$ & 0.484 \\
\hline Baseline NIHSS & 197 & $15.2(7)$ & $14.7(6.9)$ & $15.9(7.2)$ & 0.207 \\
\hline Time from onset to groin (h) & 162 & $7.9(6.1)$ & $8.35(6.54)$ & $7.03(5.05)$ & 0.19 \\
\hline IV tPA, n (\%) & 197 & $70(35.5)$ & $46(37.7)$ & $24(32)$ & 0.417 \\
\hline Proximal occlusion & 197 & $141(71.6)$ & $85(69.7)$ & $56(74.7)$ & 0.450 \\
\hline \multicolumn{6}{|l|}{ Comorbidities, n (\%) } \\
\hline $\mathrm{AF}$ & 188 & $42(22.3)$ & $29(24.8)$ & $13(18.3)$ & 0.301 \\
\hline Diabetes & 188 & $39(20.7)$ & $25(21.4)$ & $14(19.7)$ & 0.787 \\
\hline Hypertension & 188 & $124(66.0)$ & $71(60.7)$ & $53(74.6)$ & 0.051 \\
\hline \multicolumn{6}{|l|}{ Procedure } \\
\hline Time to recanalization (min) & 197 & $37.7(35.9)$ & $17.8(8.8)$ & 70 (39.8) & $<0.001$ \\
\hline Final TICI flow, n (\%) & 197 & & & & $<0.001$ \\
\hline 0 & & $1(0.5)$ & $1(0.8)$ & $0(0.0)$ & \\
\hline 1 & & $3(1.5)$ & $0(0.00)$ & $3(4)$ & \\
\hline $2 a$ & & $3(1.5)$ & $0(0.00)$ & $3(4)$ & \\
\hline $2 b$ & & $57(28.9)$ & $26(21.3)$ & $31(41.3)$ & \\
\hline $2 c$ & & $16(8.1)$ & $12(9.8)$ & $4(5.3)$ & \\
\hline 3 & & $117(59.4)$ & $83(68)$ & $34(45.3)$ & \\
\hline Final $\mathrm{TICl}$ flow, n (\%) & 197 & & & & 0.013 \\
\hline $0-2 a$ & & 7 (3.6) & $1(0.8)$ & $6(8.0)$ & \\
\hline $2 b-3$ & & $190(96.4)$ & $121(99.2)$ & $69(92.0)$ & \\
\hline Complications, n (\%) & 197 & $2(1.0)$ & $1(0.8)$ & $1(1.3)$ & 0.999 \\
\hline Hemorrhage, all types (n (\%)) & 197 & $62(31.5)$ & $29(23.8)$ & $33(44.0)$ & 0.003 \\
\hline Hemorrhage, $\mathrm{PH} 2$ & 197 & $13(6.6)$ & $3(2.5)$ & $10(13.3)$ & 0.006 \\
\hline Hemorrhage, others & 197 & $49(24.9)$ & $26(21.3)$ & $23(30.7)$ & 0.140 \\
\hline \multicolumn{6}{|l|}{ Device, n (\%) } \\
\hline Stent retrievers* & 197 & $46(23.4)$ & $16(13.1)$ & $30(40.0)$ & $<0.001$ \\
\hline Carotid stenting & 197 & $10(5.1)$ & $2(1.6)$ & 8 (11.3) & 0.007 \\
\hline Other & 197 & $143(72.6)$ & $105(86.1)$ & $38(50.7)$ & $<0.001$ \\
\hline Number of passes & 197 & & & & $<0.001$ \\
\hline 1 & & $75(38.1)$ & $60(49.2)$ & $15(20.0)$ & \\
\hline 2 & & $80(40.6)$ & $46(37.7)$ & $34(45.3)$ & \\
\hline 3 & & $35(17.8)$ & $16(13.1)$ & $19(25.3)$ & \\
\hline 4 & & 7 (3.6) & $0(0.0)$ & $7(9.3)$ & \\
\hline Number of ADAPT passes & 197 & & & & 0.115 \\
\hline 1 & & $100(50.8)$ & $68(55.7)$ & $32(42.7)$ & \\
\hline 2 & & $81(41.1)$ & 47 (38.5) & $34(45.3)$ & \\
\hline 3 & & $16(8.1)$ & $7(5.7)$ & $9(12.0)$ & \\
\hline \multicolumn{6}{|l|}{ Outcome (90 day) } \\
\hline mRS dichotomized, n (\%) & 180 & & & & $<0.001$ \\
\hline mRS $0-2$ & & $99(55)$ & $73(65.2)$ & $26(38.2)$ & \\
\hline $\mathrm{mRS}>2$ & & $81(45)$ & $39(34.8)$ & $42(61.8)$ & \\
\hline Death, n (\%) & 197 & $29(14.7)$ & $13(10.7)$ & $16(21.3)$ & 0.040 \\
\hline NIHSS (90 days) & 98 & $2.3(3.4)$ & $1.7(2.9)$ & $3.3(4.1)$ & 0.034 \\
\hline Length of stay (days) & 188 & $8.7(17.1)$ & $8.2(12.9)$ & $9.5(22.5)$ & 0.612 \\
\hline $\begin{array}{l}\text { Values are } \mathrm{n}(\%) \text { or mean (SD). } \\
\chi 2 \text { test (Fisher's exact test for sm } \\
\text { * } S \text { tent retrievers include Solitaire } \\
\text { Additionally, we dichotomized the } \\
35 \text { min ('late recan'). } \\
\text { ADAPT, a direct aspiration first pa } \\
\text { Scale; PH2, parenchymal hematon }\end{array}$ & $\begin{array}{l}\text { Student's t test for } \\
\text { tients in whom reca }\end{array}$ & ent cohort. & $\begin{array}{l}\text { 'early recan') an } \\
\text { S, modified Ran }\end{array}$ & $\begin{array}{l}\text { recanalization } \\
\text { National Instit }\end{array}$ & beyond \\
\hline
\end{tabular}

Patients in the 'late recan' group were more likely to require additional devices such as stent retrievers and carotid stents (table 1). In addition, patients in the 'late recan' group required a significantly higher number of total passes than the 'early recan' group $(\mathrm{p}<0.001)$; however, the number of ADAPT passes was not significantly different between the two groups $(p=0.115)$. The maximum number of ADAPT passes was 3 . 
Table 2 Results of the univariate and multivariable logistic regression for predictors of good outcome

\begin{tabular}{|c|c|c|c|c|c|c|c|}
\hline \multirow[b]{2}{*}{ Variable } & \multicolumn{3}{|c|}{ Univariate $(n=148)$} & \multicolumn{4}{|l|}{ Multivariate $(n=148)$} \\
\hline & OR & $95 \% \mathrm{Cl}$ & p Value & Included in model? & OR & $95 \% \mathrm{Cl}$ & p Value \\
\hline Age & 0.97 & 0.94 to 0.99 & 0.003 & Yes & 0.99 & 0.96 to 1.01 & 0.331 \\
\hline Female & 0.62 & 0.32 to 1.19 & 0.148 & Yes* & 1.44 & 0.64 to 3.25 & 0.380 \\
\hline White & 1.01 & 0.52 to 1.95 & 0.985 & Yes* & 1.03 & 0.46 to 2.27 & 0.949 \\
\hline Baseline NIHSS & 0.88 & 0.84 to 0.93 & $<0.001$ & Yes & 0.89 & 0.82 to 0.94 & $<0.001$ \\
\hline Time from onset to groin & 0.99 & 0.99 to 0.99 & 0.039 & Yes & 0.99 & 0.99 to 0.999 & 0.061 \\
\hline IV tPA & 1.10 & 0.56 to 2.15 & 0.788 & No & & & \\
\hline Groin time & 1.02 & 0.97 to 1.06 & 0.47 & No & & & \\
\hline Early recanalization ( $\leq 35 \mathrm{~min}$ ) & 2.82 & 1.41 to 5.62 & 0.003 & Yes & 0.35 & 0.15 to 0.83 & 0.017 \\
\hline Device: stent retrievers & 0.77 & 0.35 to 1.70 & 0.546 & No & & & \\
\hline Device: Precise & 1.29 & 0.30 to 5.60 & 0.999 & No & & & \\
\hline Device: other & 1.24 & 0.58 to 2.63 & 0.718 & No & & & \\
\hline Number of passes $=1$ & 1.18 & 0.52 to 2.367 & 0.401 & No & & & \\
\hline Number of passes $=2$ & 1.27 & 0.56 to 2.84 & 0.572 & No & & & \\
\hline Number of passes $>2$ & 0.54 & 0.27 to 1.07 & 0.08 & No & & & \\
\hline Post-TICI flow 'good' (2b-3) & 2.73 & 0.485 to 15.41 & 0.237 & Yes* & 0.39 & 0.05 to 3.22 & 0.384 \\
\hline $\mathrm{AF}$ & 2.04 & 0.86 to 4.81 & 0.101 & Yes* & 0.24 & 0.07 to 0.77 & 0.017 \\
\hline Diabetes & 1.00 & 0.44 to 2.24 & 0.991 & Yes* & 1.11 & 0.38 to 3.20 & 0.850 \\
\hline Hypertension & 0.83 & 0.42 to 1.61 & 0.574 & Yes* & 1.63 & 0.67 to 3.97 & 0.280 \\
\hline Waking Up & 2.82 & 0.57 to 14.1 & 0.189 & No & & & \\
\hline Complications & 0.37 & 0.03 to 4.21 & 0.408 & No & & & \\
\hline Hemorrhage & 0.38 & 0.19 to 0.76 & 0.01 & Yes & 1.75 & 0.71 to 4.31 & 0.227 \\
\hline Hemorrhage: PH2 subtype & 0.47 & 0.13 to 1.8 & 0.327 & Yes* & 1.41 & 0.25 to 7.90 & 0.697 \\
\hline
\end{tabular}

\section{Procedural complications}

Only two (1\%) patients developed intraprocedural complications other than hemorrhage-one patient from each group. One patient developed ST-segment depression with elevated troponin levels, and another patient developed non-flow limiting internal carotid artery dissection that was treated with stent placement.

Among all the patients, 31.5\% $(n=62)$ developed hemorrhage of any subtype identified on a postprocedural CT scan (table 1). Patients in the 'late recan' group were more likely to develop hemorrhage $(4.0 \%$ vs $23.8 \%, \mathrm{p}=0.003)$. The 'late recan' group had a higher likelihood of developing a $\mathrm{PH} 2$-type hemorrhage, but not other subtypes (table 1).

\section{Incidence of PH2-type hemorrhage}

The incidence of $\mathrm{PH} 2$ demonstrated a significant positive correlation with recanalization time; higher recanalization time correlated with higher incidence of $\mathrm{PH} 2$ (figure 2C). The incidence of $\mathrm{PH} 2$ was higher in the 'late recan' group than the mean $\mathrm{PH} 2$ of this study. Additionally, the mean $\mathrm{PH} 2$ rate in this sample was higher than the pooled mean from previous randomized controlled trials on endovascular stroke therapy. ${ }^{4}$ 18-21

\section{Neurological outcome}

Ninety-day mRS was used to determine the neurological outcome of patients, and these data were available for 180 patients $(91.4 \%)$ at the time of data interpretation. The overall rate of good outcome defined as an mRS score of $0-2$ at 90 days was $55 \%(n=99)$ among all patients (table 1$)$, and was higher in the 'early recan' group $(65.2 \%$ vs $38.2 \%, \mathrm{p}<0.001)$. In addition, patients in the 'late recan' group had a higher likelihood of death or being in a persistent vegetative state (mRS $5-6)(30.9 \%$ vs $15.2 \%, \mathrm{p}=0.012)$ (figure 1$)$.

The NIHSS at 90 days was available for $50 \%$ of the patients $(n=98)$ with a mean score of $2.3 \pm 3.4$. Patients in the 'early recan' group were more likely to have a lower NIHSS at 90 days (table 1). There was no difference in length of stay after the procedure between groups.

\section{Factors affecting outcome}

Baseline NIHSS, early recanalization, and atrial fibrillation were found to have a significant impact on neurological outcome measured by mRS at 90 days. A higher NIHSS score at baseline was associated with a decreased likelihood of an $\mathrm{mRS} \leq 2$ at 90 days $(\mathrm{OR}=0.89)$. The presence of atrial fibrillation was also associated with decreased likelihood of a good outcome with an OR of 0.24 (table 2). Achievement of recanalization within 35 min ('early recan') was also associated with a significant increase in the likelihood of a good outcome at 90 days with $\mathrm{OR}=2.82$. As the time to achieve recanalization increased beyond $35 \mathrm{~min}$, the percentage of patients with poor outcome at 90 days became higher than the percentage of patients with good outcomes up to $65 \mathrm{~min}$ (figure $2 \mathrm{~A}$ ).

Among patients with recanalization time $>35 \mathrm{~min}$, a subset of patients with recanalization time between 55 and $75 \mathrm{~min}$ had a nearly equal likelihood of good and poor outcomes resulting in a transient shift in the distribution curve (figure 2A). Further analysis of the characteristics of this specific subgroup showed that a significantly higher number of vessels were involved (carotid bifurcation, M1, M2, A1 ... etc) than in the 0-35 min group (more likely single vessel, ie, M1 occlusion) (figure 2B). In contrast, patients within the $35-55 \mathrm{~min}$ group showed a 
Ischemic stroke

Table 3 Factors associated with outcome grouped by recanalization time

\begin{tabular}{|c|c|c|c|c|c|}
\hline Variable & $\mathrm{N}$ & Total & $\begin{array}{l}\text { Good outcome } \\
\text { mRS } 0-2\end{array}$ & $\begin{array}{l}\text { Poor outcome } \\
\text { mRS 3-6 }\end{array}$ & $p$ Value \\
\hline \multicolumn{6}{|l|}{ Early recan } \\
\hline Age & 110 & $63.56(14.51)$ & $61.92(14.67)$ & $66.94(13.75)$ & 0.09 \\
\hline Female & 110 & $52(47.3)$ & $32(43.2)$ & $20(55.6)$ & 0.225 \\
\hline White & 110 & $62(56.4)$ & $41(55.4)$ & $21(58.3)$ & 0.771 \\
\hline Baseline NIHSS & 110 & $14.362(6.94)$ & $12.49(5.07)$ & $19.00(8.17)$ & $<0.001$ \\
\hline Onset to groin (hours) & 110 & $8.35(6.57)$ & $7.52(6.22)$ & $9.96(7.01)$ & 0.103 \\
\hline IV tPA & 110 & 40 (36.4) & $26(35.1)$ & $14(38.9)$ & 0.701 \\
\hline AF & 110 & $22(20.0)$ & $15(20.3)$ & 7 (19.4) & 0.919 \\
\hline Diabetes & 110 & $22(20.0)$ & $18(24.3)$ & $4(11.1)$ & 0.104 \\
\hline Hypertension & 110 & $74(67.3)$ & $49(66.2)$ & $25(69.4)$ & 0.735 \\
\hline Recanalization time (min) & 110 & $18.55(8.37)$ & $17.93(7.67)$ & $19.81(9.66)$ & 0.313 \\
\hline Final TICI flow & 110 & & & & 0.707 \\
\hline 0 & & $1(0.9)$ & $1(1.4)$ & $0(0.0)$ & \\
\hline 1 & & $0(0.0)$ & $0(0.0)$ & $0(0.0)$ & \\
\hline $2 a$ & & $0(0.0)$ & $0(0.0)$ & $0(0.0)$ & \\
\hline $2 b$ & & $22(20.0)$ & $14(18.9)$ & $8(22.2)$ & \\
\hline $2 c$ & & $10(9.1)$ & $8(10.8)$ & $2(5.6)$ & \\
\hline 3 & & $77(70.0)$ & $51(68.9)$ & $26(72.2)$ & \\
\hline Final TICI flow & 110 & & & & 0.484 \\
\hline $0-2 a$ & & $1(0.9)$ & $1(1.35)$ & $0(0.0)$ & \\
\hline $2 b-3$ & & 109 (99.1) & 73 (98.6) & $36(100.0)$ & \\
\hline Complication & 110 & $2(1.8)$ & $0(0.0)$ & $2(5.6)$ & 0.105 \\
\hline Hemorrhage & 110 & $25(22.7)$ & $12(16.2)$ & $13(36.1)$ & 0.019 \\
\hline Device: Trevo & 110 & $5(4.5)$ & $3(4.1)$ & $2(5.6)$ & 0.662 \\
\hline Device: Solitaire & 110 & $9(8.2)$ & $7(9.5)$ & $2(5.6)$ & 0.715 \\
\hline Device: Precise & 110 & $1(0.9)$ & $1(1.4)$ & $0(0.0)$ & 1.00 \\
\hline Device: other & 110 & $95(86.4)$ & $63(85.1)$ & 32 (88.9) & 0.59 \\
\hline \multicolumn{6}{|l|}{ Delayed recan } \\
\hline Age & 70 & $70.01(13.22)$ & 65.96 (12.84) & 72.41 (12.99) & 0.048 \\
\hline Female & 70 & $35(50.0)$ & $10(38.5)$ & $25(56.8)$ & 0.138 \\
\hline White & 70 & $43(61.4)$ & $16(61.5)$ & 27 (61.4) & 0.988 \\
\hline Baseline NIHSS & 70 & $15.96(7.42)$ & $13.12(7.38)$ & $17.36(6.99)$ & 0.013 \\
\hline Onset to groin (hours) & 70 & $7.03(5.17)$ & $6.03(4.47)$ & $7.72(5.56)$ & 0.241 \\
\hline IV tPA & 70 & $22(31.4)$ & $11(42.3)$ & $11(25.0)$ & 0.132 \\
\hline AF & 70 & $13(18.6)$ & $3(11.5)$ & $10(22.7)$ & 0.345 \\
\hline Diabetes & 70 & $13(18.6)$ & $5(19.2)$ & $8(18.2)$ & 0.913 \\
\hline Hypertension & 70 & $32(45.7)$ & $8(30.8)$ & $24(54.5)$ & 0.054 \\
\hline Recanalization time (min) & 70 & $68.51(38.91)$ & $62.81(25.30)$ & $71.89(45.01)$ & 0.349 \\
\hline Final TICI flow & 70 & & & & 0.299 \\
\hline 0 & & $0(0.0)$ & $0(0.0)$ & $0(0.0)$ & \\
\hline 1 & & $3(4.3)$ & $0(0.0)$ & $3(6.8)$ & \\
\hline $2 a$ & & $3(4.3)$ & $1(3.8)$ & $2(4.5)$ & \\
\hline $2 b$ & & $29(41.4)$ & $8(30.8)$ & $21(47.7)$ & \\
\hline $2 c$ & & $4(5.7)$ & $2(7.7)$ & $2(4.5)$ & \\
\hline 3 & & $31(44.3)$ & $15(57.7)$ & $16(36.4)$ & \\
\hline Final TICl flow & 70 & & & & 0.269 \\
\hline $0-2 a$ & & $6(8.6)$ & $1(3.8)$ & $5(11.4)$ & \\
\hline $2 b-3$ & & $64(91.4)$ & $25(96.2)$ & 39 (88.6) & \\
\hline Complication & 70 & $1(1.4)$ & $1(3.8)$ & $0(0.0)$ & 0.371 \\
\hline Hemorrhage & 70 & $29(41.4)$ & 10 (38.5) & $19(43.2)$ & 0.698 \\
\hline Device: Trevo & 70 & 13 (18.6) & $4(15.4)$ & $9(20.5)$ & 0.424 \\
\hline Device: Solitaire & 70 & $18(25.7)$ & $8(30.8)$ & $10(22.7)$ & 0.457 \\
\hline Device: Precise & 70 & $8(11.4)$ & $4(15.4)$ & $4(0.1)$ & 0.458 \\
\hline Device: other & 70 & $36(51.4)$ & $14(53.8)$ & $22(50.0)$ & 0.756 \\
\hline
\end{tabular}

Values are $\mathrm{n}(\%)$ or mean (SD).

$\chi 2$ Test (Fisher's exact test for small cell sizes) or Student's $t$ test for the entire patient cohort.

AF, atrial fibrillation; IV tPA, IV tissue plasminogen activator; mRS, modified Rankin Scale; NIHSS, National Institutes of Health Stroke Scale; TICI, Thrombolysis in Cerebral Infarction. 
lower likelihood of good outcome even with a comparable number of vessels involved in the procedure (figure $2 \mathrm{~B}$ ).

\section{Characteristics of early and late recanalization groups}

When the groups were analyzed independently based on outcome, patients with good outcome were more likely to have a lower NIHSS score at presentation than those with a poor outcome (table 3). Age was similar among patients with good and poor outcomes in the 'early recan' group, but in the 'late recan' group, patients with poor outcome were likely to be older than those with good outcome. Overall hemorrhage was more likely to be associated with a poor outcome in the 'early recan' subset of patients, but not the 'late recan' group (table 3).

\section{DISCUSSION}

In recent years, mechanical thrombectomy has been shown to significantly reduce the severity of disability after AIS with an extended treatment window. ${ }^{17-20}$ In a previous study employing older-generation thrombectomy techniques (Penumbra separator and stent retriever), ${ }^{22}$ we have shown that the duration of mechanical thrombectomy procedures is an important predictor of patient outcomes. Faster procedure time was associated with improved patient outcomes, and thrombectomies extending beyond $60 \mathrm{~min}$ increased complications and cost while diminishing the likelihood of a good outcome. ${ }^{22}$ Since that publication, new devices and techniques have been developed to improve the speed and quality of thrombectomies, such as the direct aspiration at first pass technique, or ADAPT. ${ }^{23}{ }^{24}$ With ADAPT, time to recanalization can be further reduced to, on average, $30-40$ min. $^{22} 23$

We investigated whether the relation between procedure time and outcome still exists with ADAPT. The duration of the mechanical thrombectomy procedure has been shown to be a predictor of outcome, independent of time from symptom onset to treatment, the most commonly studied and best described predictor. To evaluate the effect of procedure time in this study, patient eligibility was determined by CT perfusion imaging that defines the ischemic penumbra, and not by the time from symptom onset. In this study, we demonstrated that patients with a procedure time of $\leq 35 \mathrm{~min}$ are more likely to have a better outcome at 90 days than patients with a longer procedure time. In addition, 'late recan' was associated with decreased likelihood of achieving a good TICI flow $(\geq 2 b)$, increased likelihood of hemorrhage, specifically $\mathrm{PH} 2$, at postprocedural CT imaging, and increased use of devices, which can drive up the cost of procedure. ${ }^{22}$ Patients in the 'late recan' group had a higher incidence of PH2-type hemorrhage. We also showed a significant positive correlation between procedure time and the incidence of $\mathrm{PH} 2$, a serious complication of neuroendovascular procedures. The mean $\mathrm{PH} 2$ incidence in this study population was higher than that observed in major clinical trials in neuroendovascular therapy of $\mathrm{AIS}^{4}{ }^{18-21}$ (6.6\% vs $\left.4.3 \%\right)$. This finding is probably explained by the less stringent inclusion criteria in this study population compared with the randomized controlled trials. Patients of more advanced age, lower Alberta Stroke Program Early CT Score, and multiple intracranial occlusions as well as tandem occlusions with carotid bifurcation stenosis were included in this study sample.

Patients in the 'late recan' group required over twice as much time to achieve thrombectomy. The rationale behind using $35 \mathrm{~min}$ as the cut-off point in dichotomizing patients was that the ratio of good-to-poor outcome at increasing recanalization times is 1 at approximately $35 \mathrm{~min}$, which reflects an equal percentage of good and bad outcomes (50\%) at $35 \mathrm{~min}$ procedure time. After $35 \mathrm{~min}$, the percentage of patients with good outcomes decreases and less favorable outcomes increase. This cut-off point was determined by constructing the best-fit curve of the distribution of percentage of patients with good outcomes at different recanalization times, and then identifying the point at which this curve intersects the curve representing bad outcomes (figure 2A). It is important to note that $35 \mathrm{~min}$ does not represent an optimal procedure time, but rather represents the duration beyond which a bad outcome is more likely than a good one.

Among patients with recanalization time $>35 \mathrm{~min}$, a subset of patients with recanalization time between 55 and $75 \mathrm{~min}$ had a nearly equal likelihood of good and poor outcomes, resulting in a second inflection point in the distribution curve; however, this point is not conserved in the best-fit curves, and the higher ratio of good-to-bad outcome is not sustained beyond this one data point (figure 2A). Further analysis of the characteristics of this specific subgroup to ascertain any differences in those patients whose procedures extended to around $60 \mathrm{~min}$, showed that a significantly higher number of vessels were involved in the procedure compared with the $0-35$ min group, explaining the delay in achievement of recanalization. These patients probably represent a subset of patients with very robust collaterals with more vascular reserve that prevented penumbra progression to infarction. In contrast patients within the $35-55$ min group showed a lower likelihood of good outcome than the 0-35 min group, even with a comparable number of vessels involved in the procedure.

Patients with a procedure time longer than $35 \mathrm{~min}$ had a twofold higher likelihood of dying or being in a persistent vegetative state. While accounting for other major clinical variables that might affect neurological outcomes, longer time to recanalization was independently associated with a decreased likelihood of a good outcome at 90 days after treatment. These findings support previous studies on the influence of procedure time on outcome after thrombectomy ${ }^{22} 27$ and demonstrate that the same findings are consistent using ADAPT, with one notable exception-the primary inflection point occurs approximately 25 min earlier than with older-generation thrombectomy strategies (stent retriever and Penumbra separator). ${ }^{2}$ Thus the 'golden hour' of thrombectomy procedures is now described as the 'golden half-hour'.

Findings of this study may have implications for determination of the endpoints of mechanical thrombectomy. Although successful recanalization is associated with improved outcomes of patients, the use of multiple attempts, and thus longer procedure time, to achieve favorable angiographic outcomes is likely to be counterproductive while increasing costs. ${ }^{28}$ However, although several trials have demonstrated a negative relationship between procedure time and neurological outcome after thrombectomy, the natural history of large vessel occlusion remains so poor that including procedure time as a potential endpoint remains at the judgement of the operator.

\section{Limitations}

Patients in the 'late recan' group were older, which might have contributed to the variation in functional outcomes across the groups. However, baseline NIHSS, procedure time, and a history of atrial fibrillation, were found to be predictors of outcome on multivariate analysis, whereas age was not. A lower baseline NIHSS score was a significant predictor of good outcomes in the 'early recan' group, but not in the 'late recan' group. This is probably a surrogate for patients with poor collaterals, and underscores the importance of early recanalization in patients with a 
low NIHSS score. Although the pathophysiological processes underlying the relationship between procedure time and outcome is not fully elucidated, the likely hypothesis is that longer procedure times are associated with increased stress on collateral circulation in the penumbra, resulting in a secondary ischemic insult that accelerates the loss of salvageable penumbra.

This study is the first to evaluate the relationship between the duration of ADAPT procedure and neurological outcomes in patients with AIS. A large number of patients were enrolled from a single institution in this study, which minimizes procedural variability and disparities in perioperative patient care. Angiographic outcomes were blindly adjudicated and neurological outcomes were assessed by a stroke neurologist not involved in data collection.

\section{CONCLUSION}

Mechanical thrombectomy is increasingly used in AIS therapy and fast, safe and effective approaches such as ADAPT are continuously evolving. Our findings suggest that extending ADAPT thrombectomy procedure times beyond $35 \mathrm{~min}$ increases the risk of hemorrhage and device cost rates while worsening outcomes. These data suggest that after a time window of $35 \mathrm{~min}$ during the ADAPT procedure, it might be relevant to reassess the risks and benefits of reattempting failed thrombectomy cases.

Contributors Each author listed above should receive authorship credit based on the material contribution to this article, their revision of this article, and their final approval of this article for submission to this journal.

Competing interests Disclosures: AMS: Penumbra consulting, honorarium, speaker bureau; Pulsar Vascular consulting, honorarium, speaker bureau; Microvention consulting, honorarium, speaker bureau, research; Stryker consulting, honorarium, speaker bureau. AST, RDT, and MIC: Codman consulting, honorarium, speaker bureau, research funding; Covidien consulting, honorarium, speaker bureau; Penumbra consulting, honorarium, speaker bureau, research grants; Microvention consulting, honorarium, speaker bureau, research grants; Blockade — stock, consulting, honorarium, speaker bureau; Pulsar vascular stock, consulting, honorarium, speaker bureau, research; Medtronic consulting, honorarium, speaker bureau.

Ethics approval Medical University of South Carolina institutional review board.

Provenance and peer review Not commissioned; externally peer reviewed.

\section{REFERENCES}

1 Mozaffarian D, Benjamin EJ, Go AS, et al. Executive summary: heart disease and stroke statistics-2016 update: a report from the American Heart Association. Circulation 2016;133:447-54.

2 Tissue plasminogen activator for acute ischemic stroke. The National Institute of Neurological Disorders and Stroke rt-PA Stroke Study Group. N Engl J Med 1995;333:1581-7.

3 Nogueira RG, Lutsep HL, Gupta R, et al. Trevo versus Merci retrievers for thrombectomy revascularisation of large vessel occlusions in acute ischaemic stroke (TREVO 2): a randomised trial. Lancet 2012;380:1231-40.

4 Saver JL, Jahan R, Levy El, et al. Solitaire flow restoration device versus the Merci retriever in patients with acute ischaemic stroke (SWIFT): a randomised, parallel-group, non-inferiority trial. Lancet 2012;380:1241-9.
5 Nam J, Jing H, O'Reilly D. Intra-arterial thrombolysis vs. standard treatment or intravenous thrombolysis in adults with acute ischemic stroke: a systematic review and meta-analysis. Int I Stroke 2015;10:13-22.

6 Adams HP Jr, Adams RJ, Brott T, et al. Guidelines for the early management of patients with ischemic stroke: a scientific statement from the Stroke Council of the American Stroke Association. Stroke 2003;34:1056-83.

7 Furlan A, Higashida R, Wechsler L, et al. Intra-arterial prourokinase for acute ischemic stroke. The PROACT II study: a randomized controlled trial. Prolyse in Acute Cerebral Thromboembolism. JAMA 1999;282:2003-11.

8 Gralla J, Brekenfeld C, Mordasini P, et al. Mechanical thrombolysis and stenting in acute ischemic stroke. Stroke 2012;43:280-5.

9 Levy El, Ecker RD, Horowitz MB, et al. Stent-assisted intracranial recanalization for acute stroke: early results. Neurosurgery 2006;58:458-63; discussion -63.

10 Lin R, Vora N, Zaidi S, et al. Mechanical approaches combined with intra-arterial pharmacological therapy are associated with higher recanalization rates than either intervention alone in revascularization of acute carotid terminus occlusion. Stroke 2009:40:2092-7.

11 Lisboa RC, Jovanovic BD, Alberts MJ. Analysis of the safety and efficacy of intra-arterial thrombolytic therapy in ischemic stroke. Stroke 2002;33:2866-71.

12 Rha JH, Saver JL. The impact of recanalization on ischemic stroke outcome: a meta-analysis. Stroke 2007;38:967-73

13 Ringer AJ, Qureshi Al, Fessler RD, et al. Angioplasty of intracranial occlusion resistant to thrombolysis in acute ischemic stroke. Neurosurgery 2001;48:1282-8; discussion 1288-90

14 Smith WS, Sung G, Saver J, et al. Mechanical thrombectomy for acute ischemic stroke: final results of the Multi MERCI trial. Stroke 2008;39:1205-12.

15 Tenser MS, Amar AP, Mack WJ. Mechanical thrombectomy for acute ischemic stroke using the MERCI retriever and penumbra aspiration systems. World Neurosurg 2011;76(6 Suppl):S16-23.

16 Abou-Chebl A, Baizer CT, Krieger DW, et al. Multimodal therapy for the treatment of severe ischemic stroke combining GPIIb/llla antagonists and angioplasty after failure of thrombolysis. Stroke 2005;36:2286-8.

17 Saver JL, Goyal M, Bonafe A, et al. Stent-retriever thrombectomy after intravenous t-PA vs. t-PA alone in stroke. N Engl J Med 2015;372:2285-95.

18 Jovin TG, Chamorro A, Cobo E, et al. Thrombectomy within 8 hours after symptom onset in ischemic stroke. N Engl J Med 2015;372:2296-306.

19 Goyal M, Demchuk AM, Menon BK, et al. Randomized assessment of rapid endovascular treatment of ischemic stroke. $N$ Engl J Med 2015;372:1019-30.

20 Berkhemer $\mathrm{OA}$, Fransen PS, Beumer $\mathrm{D}$, et al. A randomized trial of intraarterial treatment for acute ischemic stroke. N Engl J Med 2015;372:11-20.

21 Campbell BC, Mitchell PJ, Kleinig TJ, et al. Endovascular therapy for ischemic stroke with perfusion-imaging selection. N Engl J Med 2015;372:1009-18.

22 Spiotta AM, Vargas J, Turner $\mathrm{R}$, et al. The golden hour of stroke intervention: effect of thrombectomy procedural time in acute ischemic stroke on outcome. J Neurointerv Surg 2014;6:511-16.

23 Turk AS, Frei D, Fiorella $D$, et al. ADAPT FAST study: a direct aspiration first pass technique for acute stroke thrombectomy. J Neurointerv Surg 2014;6:260-4.

24 Turk AS, Spiotta A, Frei D, et al. Initial clinical experience with the ADAPT technique: a direct aspiration first pass technique for stroke thrombectomy. J Neurointerv Surg 2014;6:231-7.

25 Turk AS, Nyberg EM, Chaudry MI, et al. Utilization of CT perfusion patient selection for mechanical thrombectomy irrespective of time: a comparison of functional outcomes and complications. J Neurointerv Surg 2013;5:518-22.

26 Trouillas $\mathrm{P}$, von Kummer R. Classification and pathogenesis of cerebral hemorrhages after thrombolysis in ischemic stroke. Stroke 2006;37:556-61.

27 Hassan AE, Chaudhry SA, Miley JT, et al. Microcatheter to recanalization (procedure time) predicts outcomes in endovascular treatment in patients with acute ischemic stroke: when do we stop? AJNR Am J Neuroradiol 2013:34:354-9.

28 Turk AS, Turner R, Spiotta $A$, et al. Comparison of endovascular treatment approaches for acute ischemic stroke: cost effectiveness, technical success, and clinical outcomes. J Neurointerv Surg 2015;7:666-70. 